\title{
Structural and magnetic characterization of the elusive Jahn-Teller active $\mathrm{NaCrF}_{3}$
}

\author{
Fabian L. M. Bernal $\odot$, Jonas Sottmann $\odot$, David S. Wragg ๑, and Helmer Fjellvåg $\odot$ \\ Chemistry Department and Center for Material Science and Nanotechnology, University of Oslo, Oslo NO-0315, Norway \\ Øystein S. Fjellvåg $\odot$ \\ Department for Neutron Materials Characterization, Institute for Energy Technology, PO Box 40, NO-2027 Kjeller, Norway \\ Christina Drathen* \\ ESRF- The European Synchrotron, 71, Avenue des Martyrs, Grenoble 38043, France \\ Wojciech A. Sławiński $\odot$ \\ Faculty of Chemistry, University of Warsaw, Pasteura 1, 02-093 Warsaw, Poland \\ and ISIS Facility, Rutherford Appleton Laboratory, Harwell Oxford, Didcot, Oxfordshire OX11 OQX, United Kingdom \\ Ole Martin Løvvik ๑ \\ Department of Physics, University of Oslo, Oslo NO-0315, Norway
}

(Received 11 February 2020; accepted 1 April 2020; published 15 May 2020)

\begin{abstract}
We report on the structural and magnetic properties of the elusive Jahn-Teller active compound $\mathrm{NaCrF}_{3}$, for first time synthesized in large quantities allowing detailed characterization. The crystal structure of $\mathrm{NaCrF}_{3}$ is initially described from a DFT model, which helped serve as the basis for indexing and structure determination confirmed by high-resolution synchrotron x-ray diffraction experiments. $\mathrm{NaCrF}_{3}$ adopts the triclinic space group $P \overline{1}$ (isostructural with $\mathrm{NaCuF}_{3}$ ). Magnetometry studies at low temperature show that $\mathrm{NaCrF}_{3}$ is a weak antiferromagnet, Weiss temperature $\theta=-4 \mathrm{~K}$. The Néel temperature is $T_{N}=21.3 \mathrm{~K}$ and the paramagnetic moment $\mu=4.47 \mu_{B}$ is in accordance with the theoretical $S=2$. Field-dependent measurements between 2 and $12 \mathrm{~K}$ unveil the onset of metamagnetic behavior. Our experiments revealed a weakly canted $A$-type magnetic structure observed by neutron powder diffraction, with a magnetic propagation vector $(1 / 2,1 / 2,0)$ and a magnetic moment of $3.51 \mu_{B}$ at $1.5 \mathrm{~K}$. Our results shed further light on the Jahn-Teller effects and strong correlations as a function of $A$-ion size in the family $A \mathrm{CrF}_{3}$.
\end{abstract}

DOI: 10.1103/PhysRevMaterials.4.054412

\section{INTRODUCTION}

The cooperative Jahn-Teller [1] (JT) effect is commonly ascribed to structural distortions caused by the coupling between electronically degenerate orbital states of transition-metal ions and their normal modes of vibration. This coupling results in reduction of the symmetry of the bonding environment around the JT ion to lower the total energy. JT-active perovskite-type materials are at the center of intensive research within the material science community for their wide range of physical properties and structural diversity. Superconductivity, colossal magnetoresistance (CMR), and polaron confinement are known for these compounds, giving applications in information storage and spintronics [2-4]. Perovskites have the chemical formula $A B X_{3}$. JT-active ions such as $\mathrm{Mn}^{3+}, \mathrm{Cr}^{2+}$, and $\mathrm{Cu}^{2+}$ (with electron configurations $3 d^{4}, 3 d^{4}$, and $3 d^{9}$, respectively) can occupy the octahedral $B$ site (e.g., $\left[\mathrm{MnO}_{6}\right]$ ). The octahedra are linked by their vertices forming sets of

\footnotetext{
*Present address: Bruker AXS, Öestliche Rheinbrueckenstr. 49, 76187 Karlsruhe, Germany.
}

$B-X-B$ bond angles $\xi^{\circ}$ (defined here as the perovskite angle). The electron-phonon coupling (i.e., $E \otimes e$ ) causes octahedral distortion, which favor the occupation of one of the originally degenerate orbital states. At the same time, the choice of orbital state induces an orbital ordering (OO).

The best known JT-active oxide perovskite is lanthanum manganite $\mathrm{LaMnO}_{3}$, a parent compound for several derivative crystalline compounds exhibiting CMR. An essential feature of the manganites is the role played by the atom occupying the $A$ site in influencing deformations of the perovskite-type structure, and thereby also the JT-structural distortions, leading to a rich diversity of spin, orbital, and charge orderings. In fluorides JT ions are well known for showing interesting phenomena under external stimuli. Alkali ternary manganese (III) fluorides with formula $A_{x} \mathrm{MnF}_{3+x}$ (with $A=\mathrm{Na}, \mathrm{K}, \mathrm{Cs}$ ) show significant structural diversity, adopting zero-, one-, and two-dimensional vertex-sharing arrangements of the octahedral units depending on the value of $x[5,6]$.

Three-dimensional vertex sharing high spin $3 d^{4}$ electronic configuration can form perovskite-type fluoride structures (fluoroperovskites). These include ternary chromium (II) fluoroperovskites with formula $A \mathrm{CrF}_{3}$ (where $A=$ alkali 

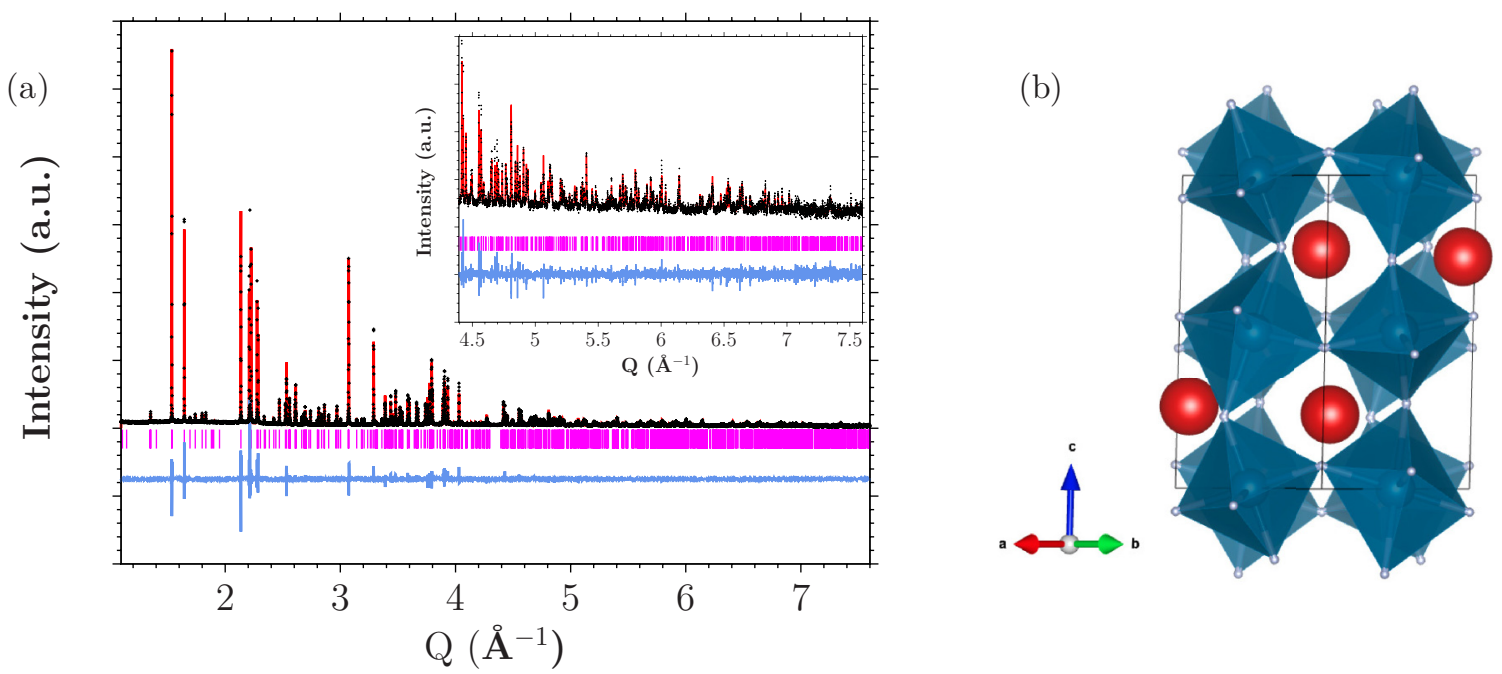

FIG. 1. (a) Final observed (black dots), calculated (red line) and difference (blue line) synchrotron x-ray powder diffraction profiles $(\lambda=0.4501 \AA)$ for $\mathrm{NaCrF}_{3}$ at $298 \mathrm{~K}\left[a=5.51515(2) \AA, b=5.68817(3) \AA, c=8.18349(3) \AA, \alpha=90.5039(3)^{\circ}, \beta=92.2554(3)^{\circ}\right.$, $\left.\gamma=86.0599(2)^{\circ}\right] . R_{w p}=11.52 \% ; R_{\exp }=5.53 \%$. Inset: Close up of the high angle region of the refined pattern. (b) Structure of $\mathrm{NaCrF}_{3}$ viewed along the [110] direction.

metals). $\mathrm{KCrF}_{3}$ has two structural-phase transitions at elevated temperatures: $I 112 / \mathrm{m} \rightarrow I 4 / \mathrm{mcm}$ at $250 \mathrm{~K}$ and $I 4 / \mathrm{mcm} \rightarrow$ $P m 3 m$ at $973 \mathrm{~K}[7,8]$, and theoretical studies have associated the metal-to-insulator transition with the onset of the tetragonal-to-cubic phase transition [9]. In addition, $\mathrm{KCrF}_{3}$ displays a rich magnetic phase diagram at low temperatures: an incommensurate antiferromagnetic ordering at $79.5 \mathrm{~K}$, an incommensurate-to-commensurate antiferromagnetic transition at $45.8 \mathrm{~K}$, and below $9.5 \mathrm{~K}$ a canted antiferromagnetic ordering with weak ferromagnetic interactions [10]. Further studies of the role played by the $A$ site in $A_{C r F}$ are currently lacking despite the interesting phase diagram of $\mathrm{KCrF}_{3}$. The main reason for this is the lack of proper synthetic protocols for the reactions of $\mathrm{Cr}^{2+}$ compounds with fluorides. The synthesis of $\mathrm{NaCrF}_{3}$ has until now proved extremely problematic due to the sensitivity of $\mathrm{Cr}^{2+}$ to oxidation. None of the synthesis routes described by Deyrup and Earnshaw et al. resulted in $\mathrm{NaCrF}_{3}$ [11,12]. To the best of our knowledge, the only evidence of the preparation of $\mathrm{NaCrF}_{3}$ was given by the work of Vollmer and UV-vis spectroscopy studies performed by Oelkrug $[13,14]$. Our new reliable synthetic protocol for $\mathrm{NaCrF}_{3}$ opens up further possibilities for synthesizing analogous materials of interest for information storage technologies, with rich states of matter and novel physical

TABLE I. Structural parameters from Rietveld refinement of HR-SPXRD data set of $\mathrm{NaCrF}_{3}$ at ambient conditions. $l, m$ and $s$ are long, medium, and short bond distances, respectively.

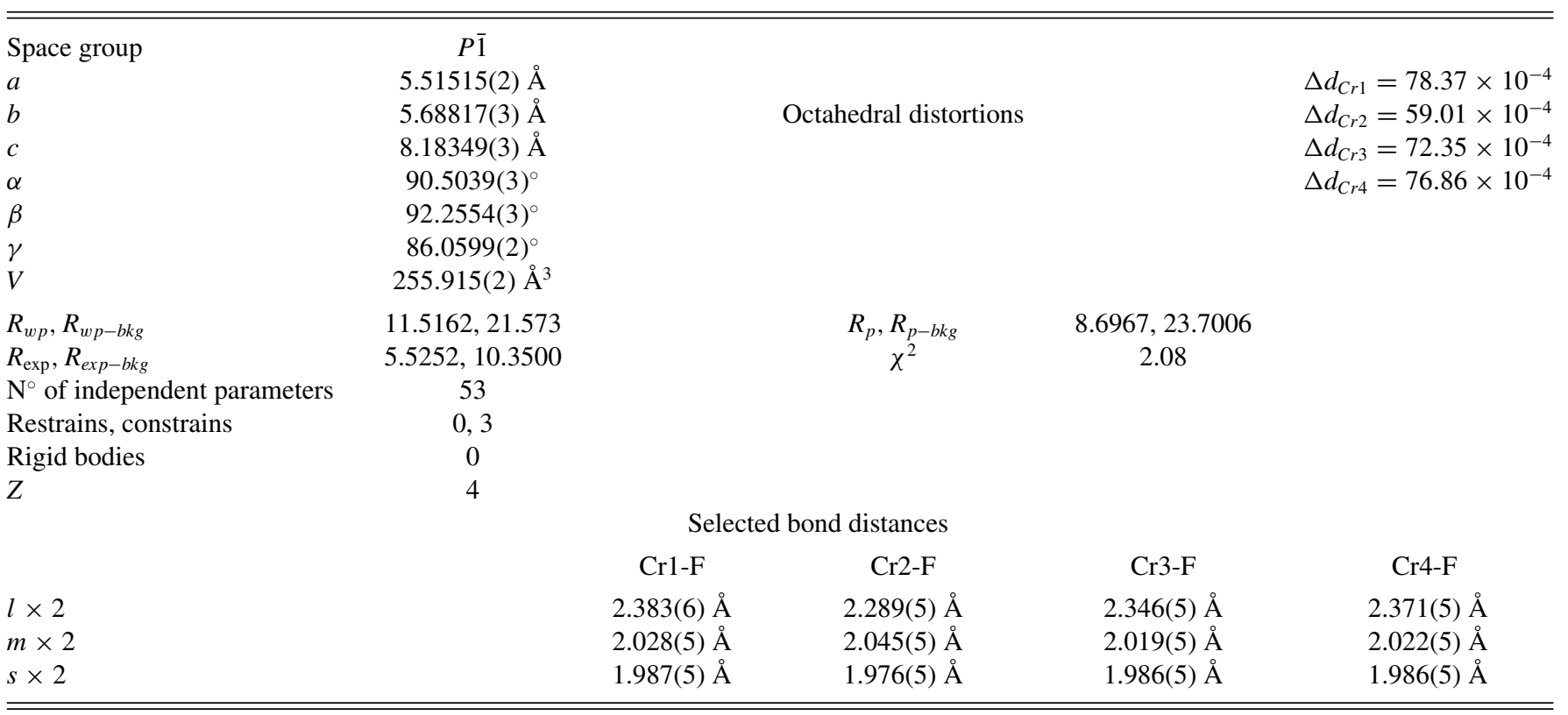


TABLE II. Parameters used to describe the magnetic structure of $\mathrm{NaCrF}_{3}$ from Rietveld refinements at $1.5 \mathrm{~K}$ in spherical coordinates with a modulation vector of $k=(1 / 2,1 / 2,0)$ in superspace group $P \overline{1}(\alpha \beta 0)$. The magnetic moment was constrained to be equal for all chromium atoms, while the polar $\varphi$ and azimuthal $\vartheta$ angles were given degrees of freedom.

\begin{tabular}{lcccc}
\hline \hline Atom Label & Atom position & Magnetic moment & Polar angle & Azimuthal angle \\
\hline $\mathrm{Cr} 1$ & $(1 / 200)$ & $M=3.519(5)$ & $\varphi_{1}=-136.99(60)$ & $\vartheta_{1}=38.9(11)$ \\
$\mathrm{Cr} 2$ & $(01 / 20)$ & $M=3.519(5)$ & $\varphi_{1}+180=43.00(60)$ & $\vartheta_{2}=129.5(8)$ \\
$\mathrm{Cr} 3$ & $(1 / 201 / 2)$ & $M=3.519(5)$ & $\varphi_{3}=-151.1(6)$ & $\vartheta_{3}=56.1(9)$ \\
$\mathrm{Cr} 4$ & $(01 / 21 / 2)$ & $M=3.519(5)$ & $\varphi_{3}+180=28.8(6)$ & $\vartheta_{4}=136.5(10)$ \\
\hline \hline
\end{tabular}

phenomena to appear in stoichiometric and nonstoichiometric modifications of the $A$ and $B$ sites in the $A \mathrm{CrF}_{3}$ family. We report for the first time the crystal and magnetic structure of the elusive JT-active compound, $\mathrm{NaCrF}_{3}$ prepared by a novel wet-chemistry method. These results are complemented by magnetometry studies.

\section{EXPERIMENTAL AND COMPUTATION SECTION}

\section{A. Synthesis of $\mathrm{NaCrF}_{3}$}

Chromium (II) acetate dihydrate $\left[\mathrm{Cr}_{2}\left(\mathrm{CH}_{3} \mathrm{CO}_{2}\right)_{4}\left(\mathrm{H}_{2} \mathrm{O}\right)_{2}\right]$ $(0.5 \mathrm{~g} 1.33 \mathrm{mmol})$ and $2 \mathrm{~mL}$ of degassed water is loaded into a $85 \mathrm{~mL}$ polycarbonate (PC) vial closed with a septum under a constant flow of Ar. $\mathrm{NaHF}_{2}(0.45 \mathrm{~g} 5.45 \mathrm{mmol})$ is dissolved in $10 \mathrm{~mL}$ deoxygenated water in a second $\mathrm{PC}$ vial under $\mathrm{Ar}$ by heating to above $50{ }^{\circ} \mathrm{C}$. The hot solution of $\mathrm{NaHF}_{2}$ is carefully and quickly injected into the vial containing $\mathrm{Cr}_{2}\left(\mathrm{CH}_{3} \mathrm{CO}_{2}\right)_{4}\left(\mathrm{H}_{2} \mathrm{O}\right)_{2}$ under vigorous stirring. $\mathrm{NaCrF}_{3}$ precipitates after few seconds. The supernatant is decanted off and the solid product is washed once with $2 \mathrm{~mL} \mathrm{50:50}$ deoxygenated water and methanol solution, and subsequently with $5 \mathrm{~mL}$ deoxygenated methanol. Finally, the product is vacuum dried overnight to yield air-stable $\mathrm{NaCrF}_{3}$.

\section{B. Computational simulations}

For the structural phase model of $\mathrm{NaCrF}_{3}$, density functional theory (DFT) was applied using the Vienna $a b$ initio simulation package $[15,16]$, with the PBE general gradient approximation (GGA) [17]. The cutoff energy of the plane wave basis set expansion was set to at least $450 \mathrm{eV}$. The density of $k$ points was determined by a maximum of $0.25 \AA^{-1}$. The structure was relaxed with remaining forces below $0.05 \mathrm{eV} / \AA$ using a quasi-Newton method.

\section{Synchrotron $x$-ray diffraction}

High-resolution synchrotron powder X-ray diffraction (HRSPXRD) experiments were conducted at ID22 beam line of the European Synchrotron (ESRF), Grenoble, France where the diffraction patterns were recorded using a wavelength of $\lambda=0.40013 \AA$ at room temperature. The crystal structure of $\mathrm{NaCrF}_{3}$ has been refined using TOPAS v5 (Bruker AXS) [18]. The initial model was obtained by DFT minimization of a symmetry free (space group $P 1$ ) triclinic model based on the crystal structure of $\mathrm{NaCuF}_{3}[13,19]$ with $\mathrm{Cr}$ replacing $\mathrm{Cu}$. This model was refined against the HR-PXRD data to obtain the correct lattice parameters and crystallite size peak broadening. The model was then processed using the ADDSYMM routine in PLATON [20] to determine the crystallographic symmetry. The new model, now in space group $P \overline{1}$, was refined against the HR-SPXRD data. Scale, lattice parameters, 13term Chebyshev polynomial background function, Gaussian crystallite size and strain, and Lorentzian strain broadening terms (fundamental parameters peak shape) and all $\mathrm{Na}$ and $\mathrm{F}$ atomic coordinates and isotropic displacement parameters were refined. Atoms of the same type ( $\mathrm{Na}, \mathrm{Cr}$, and $\mathrm{F}$ ) were constrained to have identical isotropic thermal parameters.

\section{Magnetic characterization}

Magnetometry experiments were performed on a Quantum Design 14 T Physical Property Measurement System (PPMS). Temperature-dependent dc magnetic susceptibility

TABLE III. Atomic positions of $\mathrm{NaCrF}_{3}$ from HR-SPXRD Rietveld refinement. See Table I for crystal structure details.

\begin{tabular}{|c|c|c|c|c|c|c|}
\hline Atom & Multiplicity & $\mathrm{x}$ & $\mathrm{y}$ & $\mathrm{z}$ & Occ & $U_{\text {iso }}\left(\AA^{2}\right)$ \\
\hline $\mathrm{Na} 1$ & 2 & $0.5062(8)$ & $0.5511(7)$ & $0.2370(5)$ & 1 & $0.0193(8)$ \\
\hline $\mathrm{Na} 2$ & 2 & $0.9765(8)$ & $0.0563(7)$ & $0.2603(5)$ & 1 & $0.0193(8)$ \\
\hline $\mathrm{Cr} 1$ & 1 & 0.5 & 0 & 0 & 1 & $0.0100(3)$ \\
\hline $\mathrm{Cr} 2$ & 1 & 0 & 0.5 & 0 & 1 & $0.0100(3)$ \\
\hline $\mathrm{Cr} 3$ & 1 & 0.5 & 0 & 0.5 & 1 & $0.0100(3)$ \\
\hline $\mathrm{Cr} 4$ & 1 & 0 & 0.5 & 0.5 & 1 & $0.0100(3)$ \\
\hline $\mathrm{F} 1$ & 2 & $0.6788(9)$ & $0.2832(9)$ & $0.0558(6)$ & 1 & $0.0124(6)$ \\
\hline $\mathrm{F} 2$ & 2 & $0.2019(9)$ & $0.2062(8)$ & $0.9260(6)$ & 1 & $0.0124(6)$ \\
\hline F3 & 2 & $0.7153(9)$ & $0.3238(8)$ & $0.4292(6)$ & 1 & $0.0124(6)$ \\
\hline $\mathrm{F} 4$ & 2 & $0.1887(10)$ & $0.1902(9)$ & $0.5503(7)$ & 1 & $0.0124(6)$ \\
\hline F5 & 2 & $0.3801(9)$ & $0.9405(8)$ & $0.2724(7)$ & 1 & $0.0124(6)$ \\
\hline
\end{tabular}


(a)

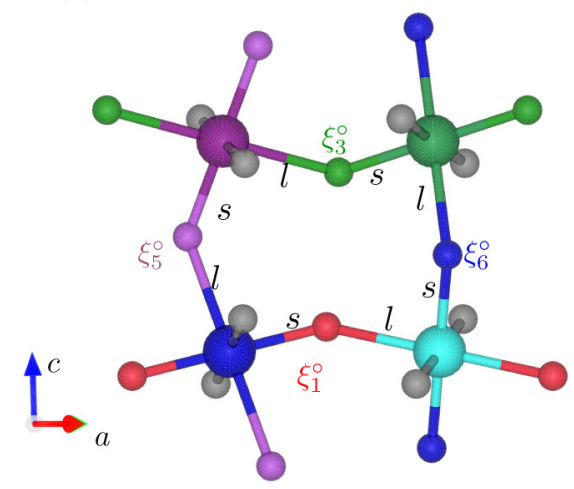

(b)

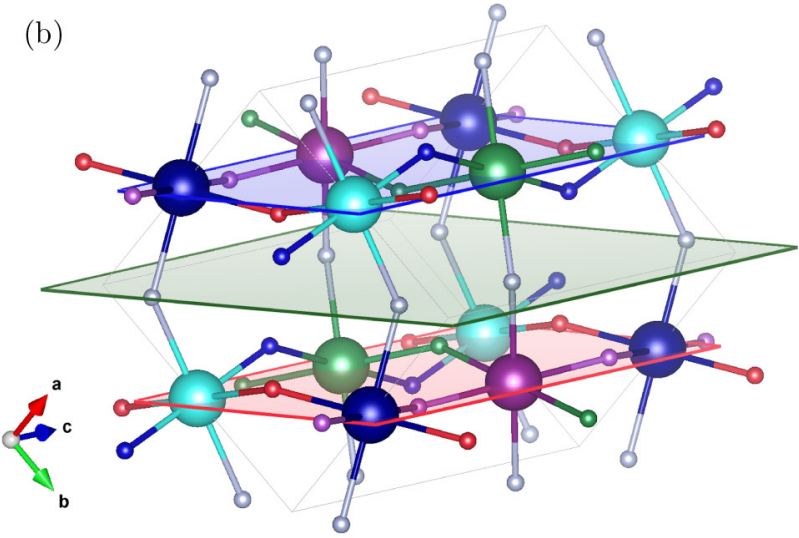

FIG. 2. (a) $l s$-bond length motif of the four crystallographic chromium sites of $\mathrm{NaCrF}_{3} . \mathrm{Cr} 1, \mathrm{Cr} 2, \mathrm{Cr} 3$, and $\mathrm{Cr} 4$ are represented here with blue, cyan, purple, and green spheres, respectively. The $\mathrm{Cr}-\mathrm{F}-\mathrm{Cr}$ bond angles are labeled as follows: $\xi_{1}^{\circ}, \mathrm{Cr} 1-\mathrm{F} 1-\mathrm{Cr} 2 ; \xi_{6}^{\circ}$, $\mathrm{Cr} 2-\mathrm{F} 6-\mathrm{Cr} 4$; $\xi_{3}^{\circ}, \mathrm{Cr} 4-\mathrm{F} 3-\mathrm{Cr} 3 ; \xi_{5}^{\circ}, \mathrm{Cr} 3-\mathrm{F} 5-\mathrm{Cr} 1$. (b) Packed crystal structure of $\mathrm{NaCrF}_{3}$ with red and blue planes marking layers of $\mathrm{Cr}^{2+}$ in which the ls-bond length motif (a) is rotated by $90^{\circ}$ relative to the adjacent layers. The midplane (green) cuts through the connecting $m$-bond distances, and represents the stacking directions of the canted antiferrodistortive orbital ordering (AOO). The unit cell is shown in pale gray.

$\chi(T)$ measurements were conducted during heating from 4$300 \mathrm{~K}$ in zero-field-cooled field-cooled mode (ZFC-FC). The magnetic susceptibility is calculated by $\chi=M / \mathrm{H}$ where $M$ is the magnetization given in emu $\mathrm{mol}^{-1}$ and the magnetic field $H=1 \mathrm{~T}$. Isothermal field-dependent measurements $[M(H)]$ were collected at $2 \mathrm{~K}$, and half-loop isothermal measurements at 4,12 , and $23 \mathrm{~K}$ up to $14 \mathrm{~T}$.

\section{E. Neutron powder diffraction}

Neutron powder diffraction (NPD) patterns were collected at ISIS Neutron and Muon Source (UK) by using the WISH long-wavelength diffractometer [21]. The sample was placed in thin wall vanadium container $(7 \mathrm{~mm}$ in diameter) and cooled down to $1.5 \mathrm{~K}$. The measurements were performed while heating from $1.5 \mathrm{~K}$ up to $127 \mathrm{~K}$ at several temperature steps. The raw data was integrated by using the MANTID suite [22] and analyzed using the JANA2006 software [23]. The structure refinement was performed using data from the four detector banks with highest resolution. The lowest resolution bank was discarded as it contained no information not present in the other detector banks. The background (10-term Chebyshev polynomial), peak-shape, isotropic thermal displacement parameters for each element type, lattice parameters and angles, and scale parameters were refined. The magnetic form factor of $\mathrm{Cr}^{2+}$ was employed in the refinements.

The superspace formalism for commensurate magnetic moment modulation was used for the magnetic structure description. Spherical coordinates were used to refine the magnetic moments. The refinements were carried out in superspace group $P \overline{1}(\alpha \beta 0)$, where [001] is the principal axis. The polar angle is the angle in the (110) plane of the magnetic moment projected in the (110) plane and [100] direction, and the azimuthal is the angle between [001] and the direction of the magnetic moment. The four $\mathrm{Cr}^{2+}$ sites were constrained to have a single magnetic moment magnitude. Polar angles $\left(\varphi_{1}\right.$ and $\varphi_{3}$ ) were refined for $\mathrm{Cr} 1$ and $\mathrm{Cr} 3$, with the polar angles of $\mathrm{Cr} 2$ and $\mathrm{Cr} 4$ constrained to values of $180+\varphi_{1}$ and $180+\varphi_{3}$ respectively. Independent azimuthal angles were refined for all $\mathrm{Cr}$ sites. These constraints are summarized in Table II. At 17 and $19 \mathrm{~K}$, the azimuthal angle of $\mathrm{Cr} 1$ and $\mathrm{Cr} 3$, and $\mathrm{Cr} 2$ and $\mathrm{Cr} 4$ were constrained to be equal. Also at $19 \mathrm{~K}$ the polar angle for $\mathrm{Cr} 1$ and $\mathrm{Cr} 3$ was fixed at values obtained at $17 \mathrm{~K}$. This is due to the low intensity of magnetic Bragg reflections near the Néel temperature and fit instability.

\section{RESULTS}

\section{A. Crystal structure determination}

To the best of our knowledge, no reliable synthesis protocol for $\mathrm{NaCrF}_{3}$ has previously been described, and the crystal structure of the compound has not been described in detail. The air sensitivity of $\mathrm{Cr}^{2+}$ is intrinsically difficult to combine with fluorine chemistry. Conventional solid-state methods are therefore unsuitable, so we developed a novel own wet-chemistry protocol. Using this we can work under conditions where $\mathrm{Cr}^{2+}$ is stable and obtain pure, single phase $\mathrm{NaCrF}_{3}$ in large quantities. We expect that other fluorides can be prepared using the same approach. Results of the Rietveld refinement against HR-PXRD data are shown in Fig. 1 and Table I. The plot, fitting statistics, and bond lengths and angles obtained indicate that the model is an excellent representation of the real structure. Table I and Table III SI show the structural parameters and atomic coordinates, as obtained from Rietveld refinements.

The $\mathrm{Cr}^{2+}$ cations occupy four nonequivalent crystallographic sites. Although the structure is triclinic, the cell edges and angles are close to those of a tetragonal unit cell. Figure 1(b) shows the crystal structure of $\mathrm{NaCrF}_{3}$ with vertex shared octahedral units (blue) with $\mathrm{Na}^{+}$ions (red) in interstices. We calculate the octahedral distortion according to the equation $\Delta_{d}=1 / 6 \sum_{n=1}^{6}\left|l_{i}-l_{a v}\right| / l_{a v}$ where $l_{i}$ are the individual bond distances of the octahedral unit, and $l_{a v}$ is the average bond distance. Figure 2(a) shows the $l$ and $s$ bonds building a tilted $l s$ motif connected through the $\mathrm{Cr}-\mathrm{F}-\mathrm{Cr}$ angles $\xi_{i}^{\circ}$. Figure 2(b) shows the $l s$ motif stacking along [110], with the bonding motif rotated $90^{\circ}$ (represented here 
TABLE IV. Selected bond angels in $\mathrm{NaCrF}_{3}$ from HR-SPXRD Rietveld refinements

\begin{tabular}{ccccc}
\hline \hline $\mathrm{F}_{i}-\mathrm{Cr}_{-} \mathrm{F}_{j}$ & $\mathrm{Cr}{ }_{(i=2,5,5 ; j=1,2,1)} \mid$ & $\mathrm{Cr}_{(i=2,1,1 ; j=6,2,6)} \mid$ & $\mathrm{Cr}{ }_{(i=4,3,3 ; j=5,4,5)} \mid$ & $90.8(2)$ \\
& $90.8(2)$ & $90.9(2)$ & $91.5(2)$ & $94.600(19)$ \\
$96.17(19)$ & $91.72(19)$ & $95.72(19)$ & $94, j=3,4,3)$ \\
\hline $94.61(19)$ & $92.95(19)$ & $94.19(19)$ & $95.50(19)$ \\
\hline \hline
\end{tabular}

as blue and red planes to indicate the $90^{\circ}$ rotation), whereas the $m$ bonds propagate above and below the (001) plane in the [110] direction. The four $\mathrm{CrF}_{6}^{4-}$ distortions can be found in Table I and selected bonds angles in Table IV. The nonequivalent octahedra are sharply tilted, corresponding to the Glazer notation $a^{-} b^{-} c^{-}$[24].

\section{B. Magnetic characterization and neutron diffraction studies}

Direct-current temperature-dependent magnetic susceptibility experiments on a polycrystalline sample of $\mathrm{NaCrF}_{3}$ between 4 and $300 \mathrm{~K}$ show a kink corresponding to the onset of long-range antiferromagnetic ordering on reaching the Néel temperature at $T_{N}=21.3 \mathrm{~K}$, Fig. 3(a). Furthermore, an upswing at around $9 \mathrm{~K}$ reveals the emergence of a weak ferromagnetic component at lower temperature. The CurieWeiss $(\mathrm{CW})$ law is applicable for the temperature range 300 $24 \mathrm{~K}$. The fit to the inverse susceptibility curves $1 / \chi$ show a linear behavior where the calculated paramagnetic moment of $\mu_{\text {eff }}=4.47 \mu_{B}$ is in reasonable agreement with the theoretical value of the spin-only configuration $S=2$ for $\mathrm{Cr}^{2+}$. The Weiss temperature is $\theta=-4 \mathrm{~K}$ measured under $1 \mathrm{~T}$ indicates just weak antiferromagnetic interactions. This contrasts with $\mathrm{KCrF}_{3}$, which displays weak ferromagnetic interactions $\theta=$ 2.7(1) $\mathrm{K}$ at $1 \mathrm{~T}$ [7]. This suggests that reduction of the ion size at the $A$ site is of paramount importance in fine tuning the magnetic exchange interactions.

Magnetic field-dependent isothermal $M(H)$ half-loops (forward and reverse field application) for $\mathrm{NaCrF}_{3}$ are presented in Fig. 3(b). These loops were measured at 2, 4, 12, and $23 \mathrm{~K}$ in applied magnetic fields up to $14 \mathrm{~T}$. At $23 \mathrm{~K}$ the half-loop shows almost linear behavior, nevertheless with a small hysteresis indicating the presence of ferromagnetic interactions. The half-loop at $12 \mathrm{~K}$ retains the hysteresis with additional signatures of metamagnetic transitions identified by a clear $\mathrm{S}$ shape occurring between 6 and $8 \mathrm{~T}$.

The metamagnetic transition becomes more pronounced with decreasing temperature as observed at 4 and $2 \mathrm{~K}$. At $4 \mathrm{~K}$ the hysteresis is at its widest. However, as shown by complete isothermal loop in the inset to Fig. 3(b), there is no longer any hysteresis at $2 \mathrm{~K}$. This means that the ferromagnetic components are suppressed by lowering the temperature. In order to identify the point of metamagnetic transition we calculated the first derivative $d \mathrm{M} / d \mathrm{H}$ of the magnetization $M$ with respect to applied field $H$ as shown in Fig. 4. An emergent peak at $8 \mathrm{~T}$ is observed below $T_{N}$ with well-defined singularities at 4 and $2 \mathrm{~K}$.

The derived synthesis protocol made it possible to prepare large-scale samples with high purity and crystallinity, well suited for detailed neutron diffraction studies. We conducted powder neutron diffraction experiments between 1.5 and $127 \mathrm{~K}$ to study the structural and magnetic changes in $\mathrm{NaCrF}_{3}$ above and below the Néel temperature. Visual inspection of the neutron diffraction patterns reveals a transition originating from the ordering of magnetic moments in the proximity of the Néel temperature, e.g., a strong reflection due to magnetic ordering occurs at $d=7.63 \AA$, Fig. 5(a). The additional magnetic reflections were indexed in a supercell with doubled $a$ - and $b$-unit cell parameters $(2 a \times 2 b \times c)$, corresponding to a propagation vector of $k=(1 / 2,1 / 2,0)$ for modulation of the magnetic structure. To describe the magnetic structure in detail, we use magnetic superspace group formalism.
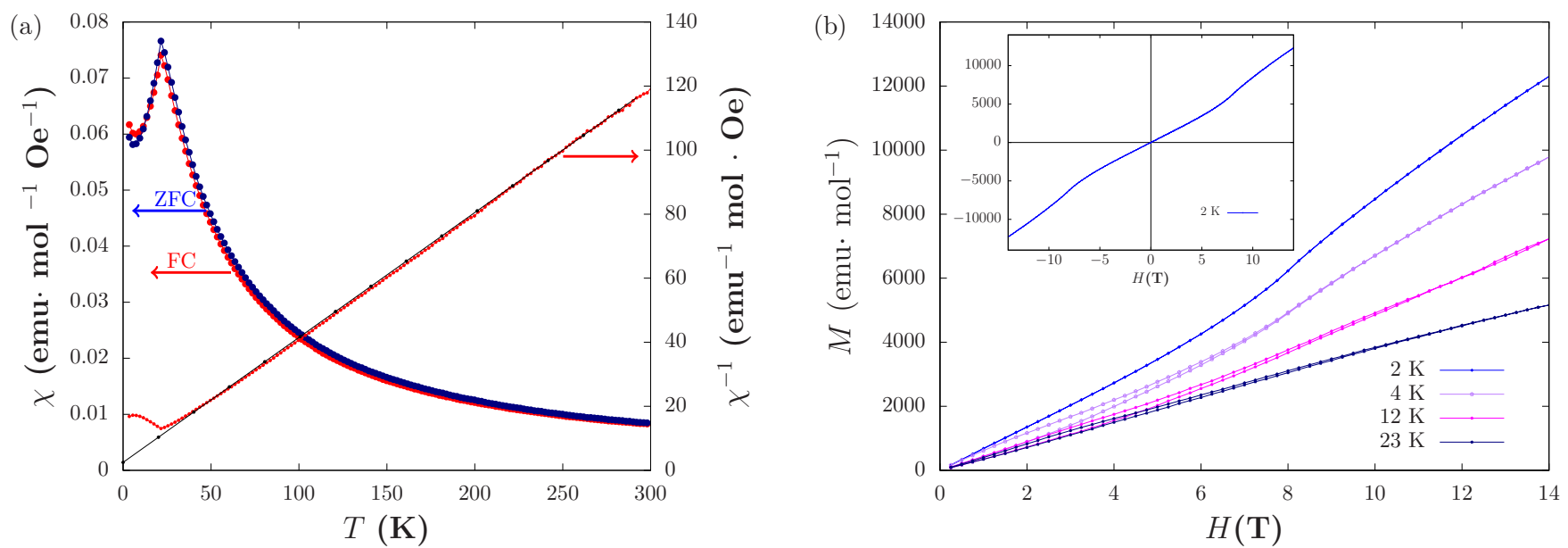

FIG. 3. (a) ZFC-FC temperature dependency of the magnetic susceptibility measured $\chi(T)$ at $H=1 \mathrm{~T}$ (left axis), and their inverse $\chi^{-1}$ (right) with the linear regression at $\theta=-4 \mathrm{~K}$. (b) Isothermal half-loop magnetization curves magnetic field $[M(H)]$ applied from $0-14 \mathrm{~T}$ and then back to $0 \mathrm{~T}$ at $2,4,12$, and $23 \mathrm{~K}$. The inset is the full $M(H)$ hysteresis loop at $2 \mathrm{~K}$ to show the symmetry at the negative quadrant. 


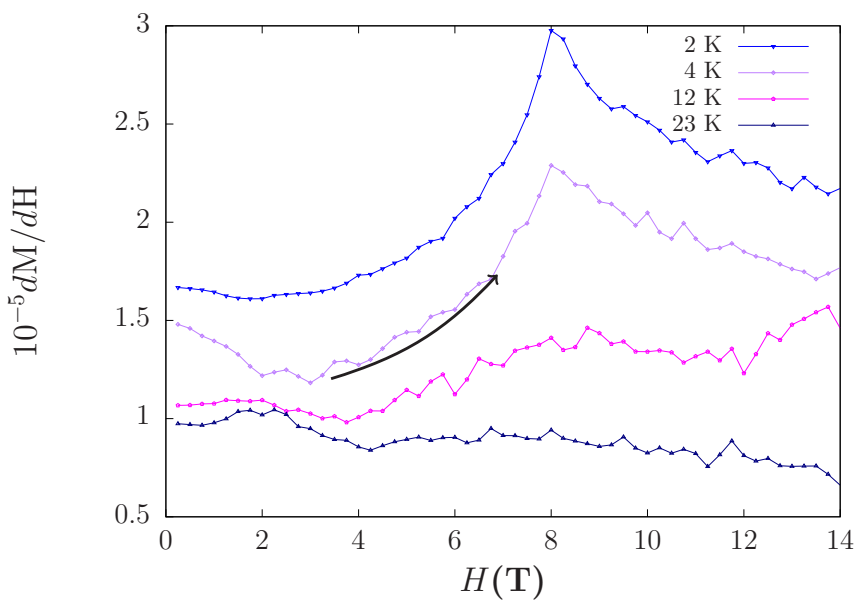

FIG. 4. First derivative $d M / d H$ of the isothermal half-loops at $2,4,12$, and $23 \mathrm{~K}$. The upswing is represented with connected line guides. A metamagnetic transition occurs at $8 \mathrm{~T}$ in the half-loops at 2 and $4 \mathrm{~K}$. Up and downswing data are emphasized by arrows for the $4 \mathrm{~T}$ data.

The magnetic structure is described in the superspace group $P \overline{1}(\alpha \beta 0)$ with a commensurate modulation vector $(1 / 2,1 / 2,0)$. There are no symmetry driven restrictions on the magnetic moment components for any of the four positions occupied by $\mathrm{Cr}$ atoms.

$\mathrm{NaCrF}_{3}$ adopts a canted $A$-type antiferromagnetic structure where chromium has an ordered magnetic moment of $\mu=$ 3.520(6) $\mu_{B}$ at $1.5 \mathrm{~K}$, Fig. 5(b). A visualization file can be found in the Supplemental Material [25]. The magnetic moments of chromium atoms are ferromagnetically ordered in the (110) planes, i.e., along [110] and [001]. We observe canting in the (110) plane. This cancels out within the magnetic unit cell due to AFM stacking along [110], which are shown by red and blue colored planes in Fig. 5. In the triclinic structure, the magnetic moments of chromium atoms point almost directly through the middle of the edge between the equatorial and axial fluorine atoms of the JT distorted $\mathrm{CrF}_{6} \mathrm{Oc}-$ tahedra. Consequently, the magnetic moments forming chains in the [111] direction. The canted $A$-type antiferromagnetic structure is in agreement with the structural $l s$ motif corresponding to ferromagnetic interactions in the (110) plane and antiferromagnetic interactions perpendicular to it. The $a^{-} b^{-} c^{-}$tilts reduces the $3 d-2 p$ overlap and weaken thereby the superexchange interactions in the presence of $\mathrm{Na}^{+}$ions.

When the direction of the magnetic moments of the four chromium sites were constrained to be either parallel or antiparallel, several weaker reflections originating from magnetic ordering at, i.e., $d=5.64$ and $5.86 \AA$ were not correctly accounted for. Therefore, we applied a slightly more complex set of constraints to the magnetic moment components. Table II presents the minimal set of magnetic structure parameters and the constraints applied in the refinement. The presence of the two reflections (at $d=5.64$ and $5.86 \AA$ ) clearly shows that the four chromium sites have slightly different canting of their magnetic moments. These subtle aspects of the magnetic structure could only be described due the high resolution and excellent signal to noise ratio of the neutron diffraction data obtained from the WISH instrument at ISIS (UK). The magnetic structure of $\mathrm{NaCrF}_{3}$ is accurately described at $1.5 \mathrm{~K}$, and details are given in Table V-VII.

The evolution of the magnetic structure was further studied below the Néel temperature $\left(T_{N}=21.3 \mathrm{~K}\right)$. In accordance with the spin only approximation $\left(\mu_{\mathrm{eff}}=4.47 \mu_{B}\right.$ in the paramagnetic regime, see above), the ordered magnetic moment of chromium is $3.520(6) \mu_{B}$ at $1.5 \mathrm{~K}$. The slightly lower experimental value compared to the theoretical value (of $4 \mu_{B}$ ) is attributed to hybridization in the chemical bonding, which effectively reduces the number of electrons contributing to the magnetic moment. The ordered magnetic moment steadily decreases from $\mu=3.520(6) \mu_{B}$ at $1.5 \mathrm{~K}$ with increasing temperature up to the Néel temperature at $21 \mathrm{~K}$ where the magnetic ordering disappears [see Fig. 6(a)].
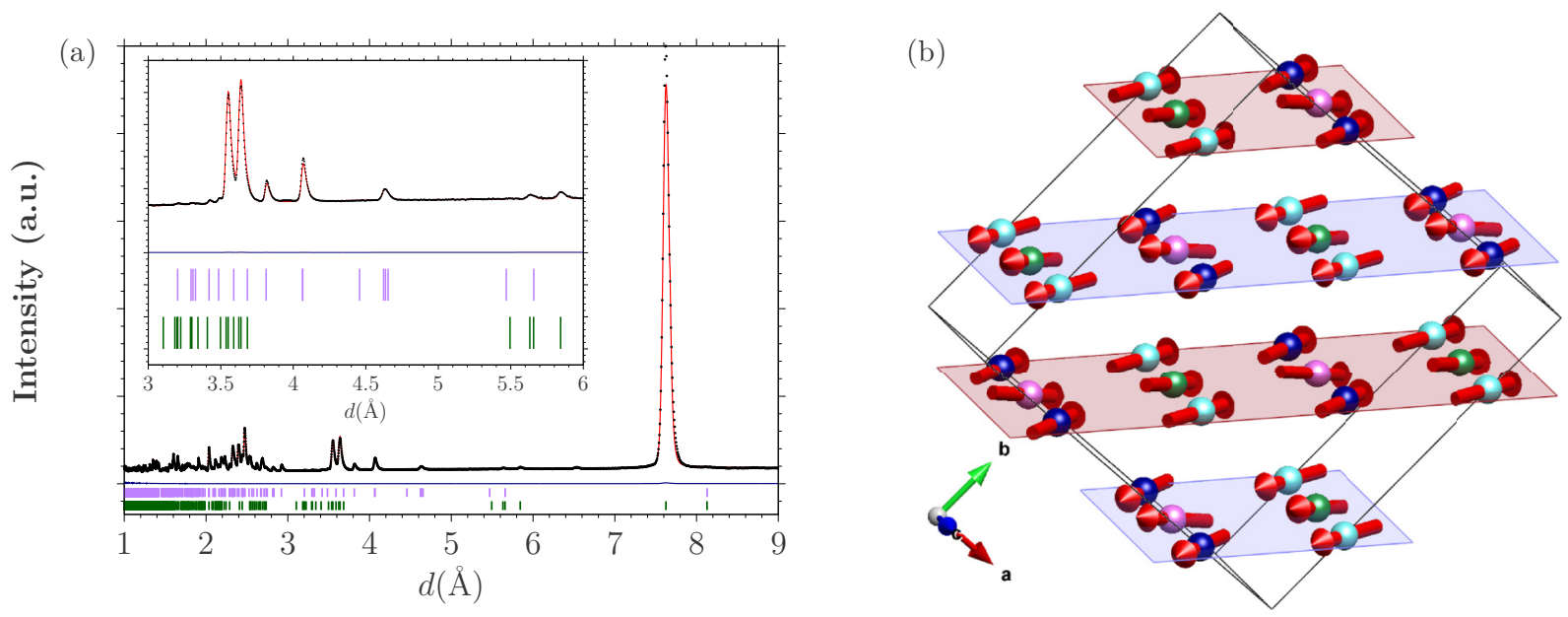

FIG. 5. (a) Rietveld refinements of NPD data set of $\mathrm{NaCrF}_{3}$ at $1.5 \mathrm{~K}$ from detector bank 2 (lowest resolution bank used in refinements) showing the peak at $7.63 \AA$ with the inlet showing small peaks of the magnetic phase. The purple and green tics correspond to the crystal and magnetic phase, respectively. (b) Magnetic structure of $\mathrm{NaCrF}_{3}$ in the $[1,-1,0]$ direction. The antiparallel alignment of the spins is represented by the blue-red sequence. Blue, cyan, purple, and green atoms correspond to $\mathrm{Cr} 1, \mathrm{Cr} 2, \mathrm{Cr} 3$, and $\mathrm{Cr} 4$ respectively. 
TABLE V. Structural parameters from Rietveld refinement of NPD dataset of $\mathrm{NaCrF}_{3}$ at $1.5 \mathrm{~K} . l, m$ and $s$ are long, medium and short bond distances, respectively.

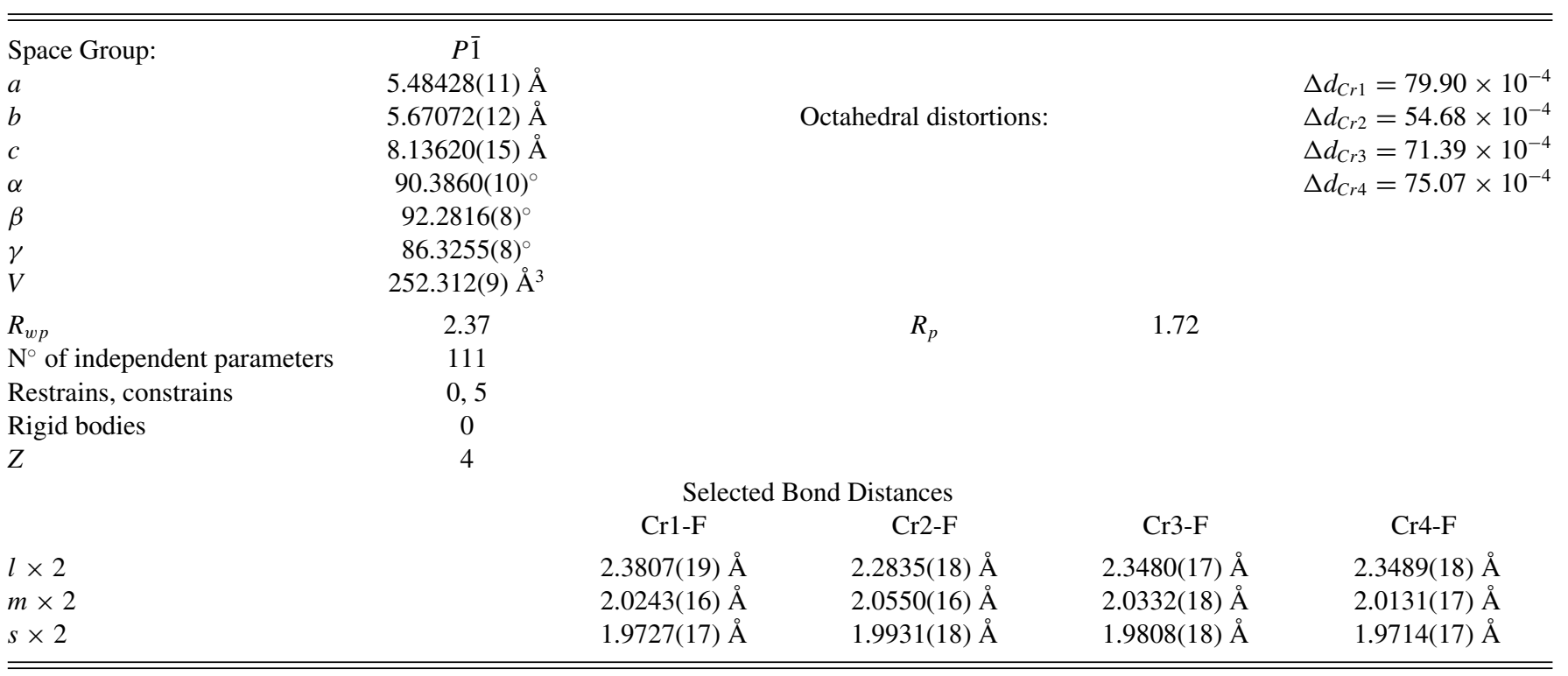

The polar angle difference between the magnetic moments of $\mathrm{Cr} 1$ and $\mathrm{Cr} 3$ is fairly constant [see Figs. 6(b), 6(c)]. The two pairs of azimuthal angles $(\mathrm{Cr} 1, \mathrm{Cr} 3)$ and $(\mathrm{Cr} 2, \mathrm{Cr} 4)$ show similar values, but cannot be constrained to become equal without worsening the fit. However, at 17 and $19 \mathrm{~K}$ the azimuthal angle $\vartheta$ of $\mathrm{Cr} 1$ and $\mathrm{Cr} 3$, and $\mathrm{Cr} 2$ and $\mathrm{Cr} 4$ could successfully be constrained. For the at $19 \mathrm{~K}$ data all angle values were frozen at values obtained at $17 \mathrm{~K}$ due to fit instability. The antiferromagnetic ordering at the Néel temperature is associated with a significant thermal contraction of the lattice upon cooling, Fig. 7. At the ordering temperature, changes in the tilting of the octahedra is revealed by the analyzed changes in the perovskite bond angles. These observations indicate a clear magnetostructural coupling in $\mathrm{NaCrF}_{3}$.

\section{DISCUSSION}

The reliable new synthesis route for $\mathrm{NaCrF}_{3}$ allowed us to undertake a detailed study of its structure and magnetic prop-

TABLE VI. Atomic positions of $\mathrm{NaCrF}_{3}$ from NPD Rietveld refinements at $1.5 \mathrm{~K}$. See Table $\mathrm{V}$ for crystal structure details.

\begin{tabular}{lcccccc}
\hline \hline Atom & Multiplicity & $\mathrm{x}$ & $\mathrm{y}$ & $\mathrm{z}$ & Occ & $U_{\text {iso }}\left(\AA^{2}\right)$ \\
\hline $\mathrm{Na} 1$ & 2 & $0.5084(5)$ & $0.5551(4)$ & $0.2358(3)$ & 1 & $0.0181(4)$ \\
$\mathrm{Na} 2$ & 2 & $0.9718(5)$ & $0.0597(4)$ & $0.2621(3)$ & 1 & $0.0181(4)$ \\
$\mathrm{Cr} 1$ & 1 & 0.5 & 0 & 0 & 1 & $0.0085(4)$ \\
$\mathrm{Cr} 2$ & 1 & 0 & 0.5 & 0 & 1 & $0.0085(4)$ \\
Cr3 & 1 & 0.5 & 0 & 0.5 & 1 & $0.0085(4)$ \\
Cr4 & 1 & 0 & 0.5 & 0.5 & 1 & $0.0085(4)$ \\
F1 & 2 & $0.6791(3)$ & $0.2814(3)$ & $0.0585(2)$ & 1 & $0.0151(3)$ \\
F2 & 2 & $0.2023(3)$ & $0.2052(3)$ & $0.9229(2)$ & 1 & $0.0151(3)$ \\
F3 & 2 & $0.7161(3)$ & $0.3249(3)$ & $0.4261(2)$ & 1 & $0.0151(3)$ \\
F4 & 2 & $0.1854(4)$ & $0.1900(3)$ & $0.5525(2)$ & 1 & $0.0151(3)$ \\
F5 & 2 & $0.3750(3)$ & $0.9405(2)$ & $0.2727(2)$ & 1 & $0.0151(3)$ \\
F6 & 2 & $0.1252(3)$ & $0.4358(3)$ & $0.2297(2)$ & 1 & $0.0151(3)$ \\
\hline \hline
\end{tabular}

erties for the first time. The JT-active ions $\mathrm{Cr}^{2+}$ of $\mathrm{NaCrF}_{3}$ occupy four nonidentical crystallographic sites with different octahedral distortions. Our results demonstrate the importance of the ion size at the $A$ site in tuning the properties of the JT-active $B$-site ions. $A$-site-dependent physical phenomena have previously been observed in the $d^{4}$ isoelectronic lowdimensional manganese (III) fluoroperovskites, where variations in the $A$-site ion size give rise to rich and interesting phase diagrams under external stimuli.

A significant feature of $\mathrm{NaCrF}_{3}$ is its metamagnetic signatures below $T_{N}$ under field-dependent measurements in addition to weak residual ferromagnetic interactions at $23 \mathrm{~K}$. The presence of metamagnetism in $\mathrm{NaCrF}_{3}$ resembles in some aspects other known systems with exotic properties (see Refs. [26-28]). We believe that this behavior is related to correlations between the orbital structure and magnetic ordering as discussed by Kugel and Khomskii [29]. The temperature-dependent NPD data reveals a smooth decrease in the unit cell volume and $\gamma$ angle above $T_{N}$, with a rapid collapse at lower temperatures, Fig. 7(a). One would expect that the $\xi^{\circ}$ angle would reduce for all four $\mathrm{Cr}^{2+}$ sites, however, they follow independent patterns as shown in Fig. 7(b). $\xi_{6}^{\circ}$ displays a slight decrease upon cooling while $\xi_{1}^{\circ}$ increases. Perovskite angle reduction further decreases the orbital overlap, weakening the magnetic interactions while reinforcing Cr-to-Cr interactions. The refined magnetic moments of $\mathrm{Cr}^{2+}$ ions in $\mathrm{NaCrF}_{3}$ are in agreement with NPD studies on $\mathrm{KCrF}_{3}$ by Xiao et al. [10]. Compared to other sodium transition-metal fluoroperovskites, $\mathrm{NaCrF}_{3}$ deviates from the family trend by displaying a canted $A$-type magnetic ordering compared to the $G$ types found in $\mathrm{NaNiF}_{3}$ and $\mathrm{NaCoF}_{3}[30,31]$.

To further investigate the role of the $A$ site in $A \mathrm{CrF}_{3}$ we report elsewhere the use of UV-vis spectroscopy along with magnetic characterization studies to compare the local electronic structure of the JT systems $\mathrm{KCrF}_{3}$ and $\mathrm{NaCrF}_{3}$ as a function of temperature and magnetic field [32]. Such experiments could provide more detailed information on the 
TABLE VII. Magnetic parameters of $\mathrm{Cr}^{2+}$ ions in $\mathrm{NaCrF}_{3}$ from Rietveld refinements of PND as function of temperature. At $17 \mathrm{~K}$ the azimuthal angle is restricted to be equal for $\mathrm{Cr} 1$ and $\mathrm{Cr} 3$, and $\mathrm{Cr} 2$ and $\mathrm{Cr}$. The polar and azimuthal angles are not refined for the $19 \mathrm{~K}$ data set, but restricted to be equal to the refined values from the $17 \mathrm{~K}$ data set.

\begin{tabular}{|c|c|c|c|c|c|c|c|c|c|}
\hline Temperature & M & $\varphi(\mathrm{Cr} 1)$ & $\vartheta(\mathrm{Cr} 1)$ & $\varphi(\mathrm{Cr} 2)$ & $\vartheta(\mathrm{Cr} 2)$ & $\varphi(\mathrm{Cr} 3)$ & $\vartheta(\mathrm{Cr} 3)$ & $\varphi(\mathrm{Cr} 4)$ & $\vartheta(\mathrm{Cr} 4)$ \\
\hline 1.5 & $3.519(5)$ & $-136.99(60)$ & $38.9(11)$ & $43.00(60)$ & $129.5(8)$ & $-151.1(6)$ & $56.1(9)$ & $28.8(6)$ & $136.5(10)$ \\
\hline 5 & $3.466(6)$ & $-137.13(80)$ & $39.5(13)$ & $42.86(80)$ & $129.5(10)$ & $-149.6(9)$ & $55.5(10)$ & $30.3(9)$ & $136.3(11)$ \\
\hline 7 & $3.405(5)$ & $-136.60(80)$ & $38.3(17)$ & $43.39(80)$ & $128.9(14)$ & $-149.7(9)$ & $54.4(14)$ & $30.2(9)$ & $134.9(16)$ \\
\hline 9 & $3.291(5)$ & $-136.7(13)$ & $39.7(13)$ & $43.2(13)$ & 133.1(11) & $-149.2(14)$ & $55.9(11)$ & $30.7(14)$ & $136.6(11)$ \\
\hline 11 & $3.128(5)$ & $-135.8(10)$ & $42.1(11)$ & $44.1(10)$ & $134.9(9)$ & $-150.2(11)$ & $57.6(9)$ & $29.7(11)$ & 139.0(9) \\
\hline 13 & $2.900(5)$ & $-133.40(80)$ & $36.7(29)$ & $46.59(80)$ & $130.0(24)$ & $-151.4(9)$ & $52.1(24)$ & $28.5(9)$ & $134.7(28)$ \\
\hline 15 & $2.576(5)$ & $-131.54(80)$ & $40.3(19)$ & $48.45(80)$ & $133.1(16)$ & $-154.1(9)$ & $53.4(16)$ & $25.8(9)$ & $136.7(17)$ \\
\hline 17 & $2.023(5)$ & $-129.6(10)$ & 49.1(14) & $50.3(10)$ & $137.5(13)$ & $-158.6(10)$ & 49.1 & $21.3(10)$ & 137.5 \\
\hline 19 & $0.612(7)$ & -129.6 & 49.1 & 50.3 & 137.5 & -158.6 & 49.1 & 21.3 & 137.5 \\
\hline
\end{tabular}

strength of the JT distortions and be used to assess OO melting points in JT-active fluorides.

\section{CONCLUSIONS}

This work provides compelling evidence of the existence of the JT-active compound $\mathrm{NaCrF}_{3}$, and describes its structural and magnetic properties. The successful development of a reliable and reproducible synthesis route, provided the required materials basis for shedding more light on the properties of the $A \mathrm{CrF}_{3}$ family, which previously proved elusive owing to the air-sensitive chemistry of $\mathrm{Cr}^{2+}$. The structural and magnetic phase diagram of $\mathrm{NaCrF}_{3}$ is much simpler than the diverse situation observed for $\mathrm{KCrF}_{3}$ at low temperature. This is due to the smaller $A$-ion size causing the $\mathrm{NaCrF}_{3}$ structure to adopt the low symmetry space group $P \overline{1}$ at relatively high temperature. The low symmetry structure is responsible

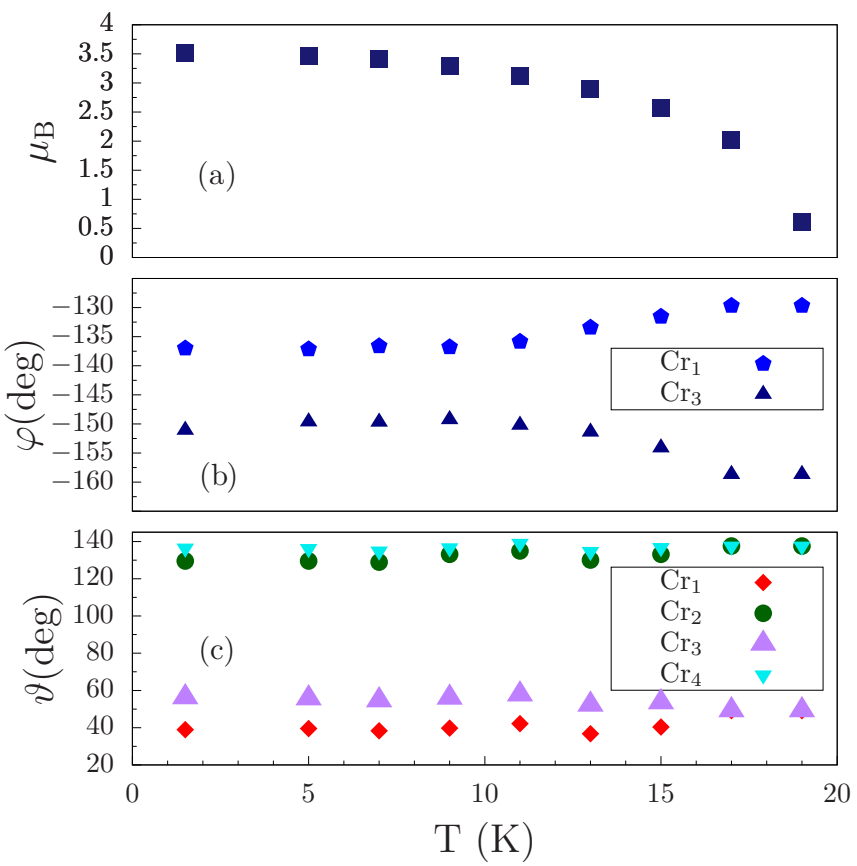

FIG. 6. (a) Magnetic moment of the chromium cations in $\mathrm{NaCrF}_{3}$ determined by neutron diffraction as a function of temperature. Temperature evolution of the (b) polar and (c) azimuthal angles. Constraints are described in Table II. for the unusual metamagnetic behavior of $\mathrm{NaCrF}_{3}$, which can be clearly linked to variations in both the crystal structure (perovskite angles and lattice parameters) and the magnetic structure (polar and azimuthal angles of the magnetic

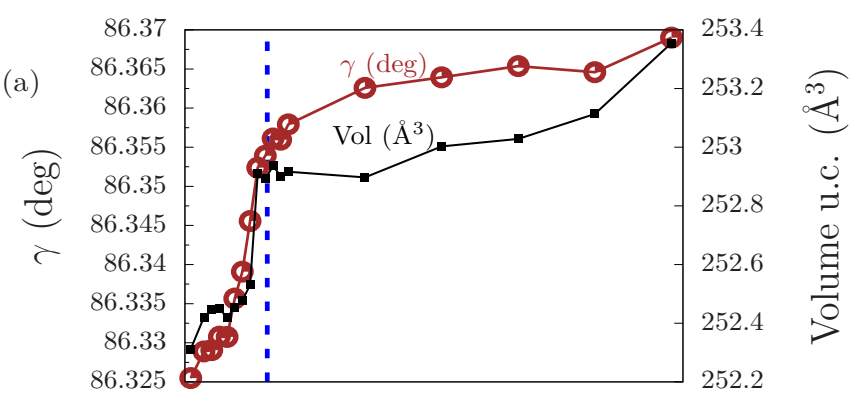

(b)

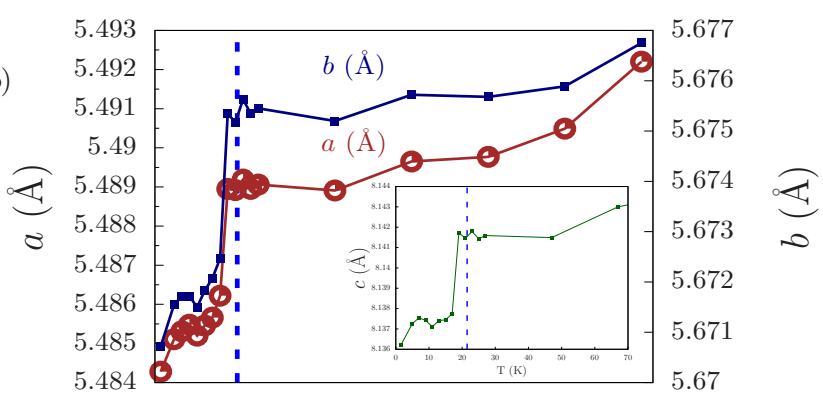

(c)

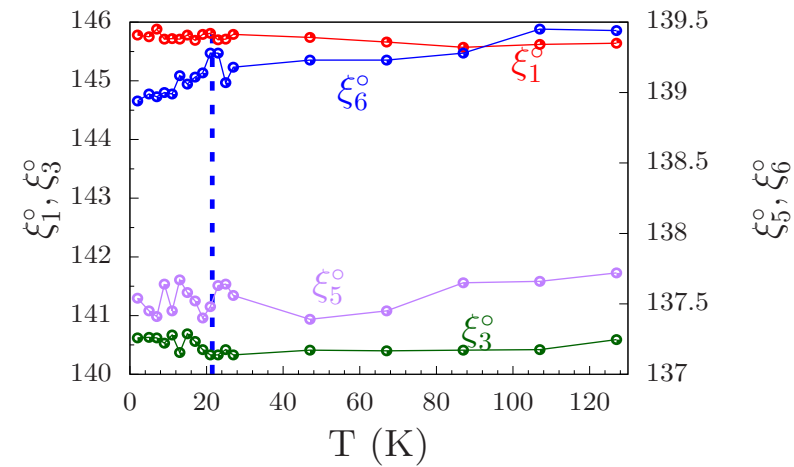

FIG. 7. (a) Temperature dependence of unit cells dimensions $(a, b, \gamma, \mathrm{V})$ and $\xi^{\circ}$ angles. (b) Temperature dependence of the unit cell lattice parameters (c) Perovskite angles composing the canted $l s$ motif $\mathrm{NaCrF}_{3}$ as function of temperature: $\xi_{1}^{\circ}=\mathrm{Cr} 1-\mathrm{F} 1-\mathrm{Cr} 2, \quad \xi_{6}^{\circ}=\mathrm{Cr} 2-\mathrm{F} 6-\mathrm{Cr} 4, \quad \xi_{3}^{\circ}=$ $\mathrm{Cr} 4-\mathrm{F} 3-\mathrm{Cr} 3, \xi_{5}^{\circ}=\mathrm{Cr} 3-\mathrm{F} 5-\mathrm{Cr} 1$. Vertical dashed line at $T_{N}$ to emphasize the place where the magnetic long-range order sets in. 
moments), observed in the variable temperature NPD data. The new synthesis protocol opens up the possibility of preparing numerous novel stoichiometric compounds by tuning the $A$ and $B$ sites in fluoroperovskites, which in turn may reveal new and interesting physical properties.

\section{ACKNOWLEDGMENTS}

We thank Serena Margadonna (Swansea University, Swansea, UK) for granted financing support by the Norwe- gian Research Council (Norges Forskningsråd NFR) project 214260. The U.K. Science and Technology Facilities Council (STFC) is thanked for allocating beam time at the ISIS Facility. We also thank Pascal Manuel for help during the experiment. We aknowldge CRISMAT laboratory (Caen France) for the magnetization measurements up to $14 \mathrm{~T}$ and Fabien Veillon and Bruno Gonano for technical and analysis help. We thank Susmit Kumar for discussions.
[1] H. A. Jahn and E. Teller, Proc. R. Soc A 161, 220 (1937).

[2] H. M. Rønnow, Ch. Renner, G. Aeppli, T. Kimura, and Y. Tokura, Nature (London) 440, 1025 (2006).

[3] J. M. De Teresa, M. R. Ibarra, P. Algarabel, L. Morellon, B. García-Landa, C. Marquina, C. Ritter, A. Maignan, C. Martin, B. Raveau, A. Kurbakov, and V. Trounov, Phys. Rev. B 65, 100403(R) (2002).

[4] G. Alvarez, M. Mayr, A. Moreo, and E. Dagotto, Phys. Rev. B 71, 014514 (2005).

[5] F. Rodriguez and F. Aguado, J. Chem. Phys 118, 10867 (2003).

[6] F. Aguado, F. Rodriguez, and P. Núñez, Phys. Rev. B 76, 094417 (2007).

[7] S. Margadonna and G. Karotsis, J. Amer. Chem. Soc. 128, 16436 (2006).

[8] S. Margadonna and G. Karotsis, J. Mater Chem 17, 2013 (2007).

[9] G. Wang, Z. Li, L. Zheng, and Z. Yang, Phys. Rev. B 84, 045111 (2011).

[10] Y. Xiao, Y. Su, H. F. Li, C. M. N. Kumar, R. Mittal, J. Persson, A. Senyshyn, K. Gross, and Th. Brueckel, Phys. Rev. B 82, 094437 (2010).

[11] A. J. Deyrup, Inorg. Chem 3, 1645 (1964).

[12] A. Earnshaw, L. F. Larkworthy, and K. S. Patel, J. Chem. Soc. A 363 (1966).

[13] G. Vollmer, Untersuchungen an ternären Fluoriden $\mathrm{AMeF}_{3}$ un $\mathrm{AMeF}_{4}, \mathrm{Ph} . \mathrm{D}$. thesis, University of Tübingen, Germany, 1966.

[14] V. D. Oelkrug, Berichte der Bunsengesellschaft für physikalische Chemie 70, 737 (1966).

[15] G. Kresse and J. Furthmuller, Phys. Rev. B 54, 11169 (1996).

[16] G. Kresse and J. Hafner, Phys. Rev. B 47, 558 (1997).

[17] J. P. Perdew, K. Burke, and M. Ernzerhof, Phys. Rev. Lett. 77, 3865 (1996).

[18] A. Coelho, J. Appl. Cryst. 51, 210 (2018).
[19] V. Keiser, M. Otto, F. Binder, and D. Babel, Z. Anorg. Allg. Chem. 585, 93 (1990).

[20] A. L. Spek, Acta Crystallogr. D D65, 148 (2009).

[21] L. C. Chapon and P. Manuel, Neutron News 22, 22 (2011)

[22] O. Arnold, J. C. Bilheux, J. M. Borreguero, A. Buts, S. I. Campbell, L. Chapon, M. Doucet, N. Draper, R. Ferraz Leal, M. A. Gigg, V. E. Lynch, A. Markvardsen, D. J. Mikkelson, R. L. Mikkelson, R. Miller, K. Palmen, P. Parker, G. Passos, T. G. Perring, P. F. Peterson, S. Ren, M. A. Reuter, A. T. Savici, J. W. Taylor, R. J. Taylor, R. Tolchenov, W. Zhou, and J. Zikovsky, Nucl. Instrum. Methods Phys. Res. A 764, 156 (2014).

[23] V. Petricek, M. Dusek, and L. Palatinus, Z. Kristallogr. Cryst. Mater 229, 345 (2014)

[24] A. M. Glazer, Acta Cryst. B28, 3384 (1972).

[25] See Supplemental Material at http://link.aps.org/supplemental/ 10.1103/PhysRevMaterials.4.054412 for VESTA file visualization of magnetic structure.

[26] N. Matsubara, C. Martin, B. Vertruyen, A. Maignan, F. Fauth, P. Manuel, V. Hardy, D. Khalyavin, E. Elkaim, and F. Damay, Phys. Rev. B 100, 014409 (2019).

[27] Y. Zhou, X. Zhu, S. Huang, X. Chen, Y. Zhou, C. An, B. Zhang, Y. Yuan, Z. Xia, C. Gu, and Z. Yang, Phys. Rev. B 96, 205122 (2017).

[28] J. P. Bolletta, F. Pomiro, R. D. Sanchez, V. Pomjakushin, G. Aurelio, A. Maignan, C. Martin, and R. E. Carbonio, Phys. Rev. B 98, 134417 (2018).

[29] K. I. Kugel and D. I. Khomskii, Sov. Phys. Usp 25, 231 (1982).

[30] A. Epstein, J. Makovsky, M. Melamud, and H. Shaked, Phys. Rev. 174, 560 (1968).

[31] Z. Friedman, M. Melamud, J. Makovsky, and H. Shaked, Phys. Rev. B 2, 179 (1970).

[32] F. L. M. Bernal, F. Lundvall, S. Kumar, P. A. Hansen, D. S. Wragg, O. M. Løvvik, and H. Fjellvåg (unpublished). 